\title{
Alternative coulometric signal readout based on a solid-contact ion- selective electrode for detection of nitrate
}

\author{
Hemin Wang ${ }^{\text {a, b }}$, Baiqing Yuan ${ }^{\text {a, }}{ }^{* *}$, Tanji Yin ${ }^{\text {b, c, d, * }}{ }^{\text {, Wei Qin }}{ }^{\text {b, c, d }}$ \\ a School of Chemistry and Materials Science, Ludong University, Yantai, 264025, Shandong, PR China \\ ${ }^{\mathrm{b}}$ Key Laboratory of Coastal Environmental Processes and Ecological Remediation, Yantai Institute of Coastal Zone Research (YIC), Chinese Academy of \\ Sciences (CAS), Shandong Provincial Key Laboratory of Coastal Environmental Processes, YICCAS, Yantai, Shandong, 264003, PR China \\ ${ }^{\mathrm{c}}$ Laboratory for Marine Biology and Biotechnology, Pilot National Laboratory for Marine Science and Technology, Qingdao, 26620, PR China \\ ${ }^{\mathrm{d}}$ Center for Ocean Mega-Science, Chinese Academy of Sciences, Qingdao, Shandong, 266071, PR China
}

\section{H I G H L I G H T S}

- A coulometric signal readout is proposed based on OMC-based solidcontact $\mathrm{NO}_{3}^{-}$-ISE.

- The signal depends on OMC with electrical double layer capacitance as solid contact.

- The coulometric signal for $\mathrm{NO}_{3}^{-}$-ISE is robust to $\mathrm{O}_{2}, \mathrm{CO}_{2}$ and light interferences.

\section{A R T I C L E I N F O}

\section{Article history:}

Received 1 June 2020

Received in revised form

6 July 2020

Accepted 11 July 2020

Available online 23 July 2020

\section{Keywords:}

Coulometric signal

Solid-contact ion-selective electrode

Electrical double layer capacitance

Ordered mesoporous carbon

$\mathrm{NO}_{3}^{-}$
G R A P H I C A L A B S T R A C T

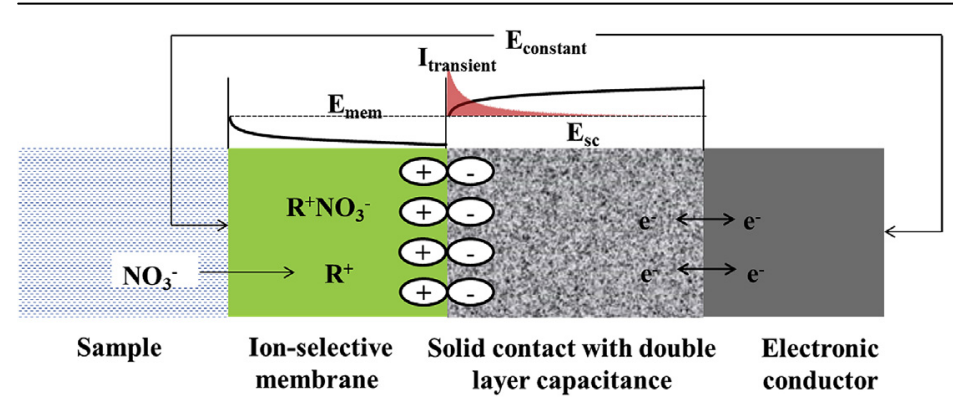

\begin{abstract}
A B S T R A C T
Traditional potentiometric $\mathrm{NO}_{3}^{-}$-selective electrodes suffer from a fundamental limitation of the Nernst slope $\left(59.1 \mathrm{mV} / \mathrm{dec}\right.$ at $\left.25^{\circ} \mathrm{C}\right)$ due to the relationship between the potential and the logarithmic of ionic activity. Herein, a coulometric signal readout is proposed instead of the potentiometric response for detection of $\mathrm{NO}_{3}^{-}$based on an ordered mesoporous carbon (OMC)-based solid-contact ion-selective electrode (ISE). The mechanism for obtaining the coulometric signal is based on the electrical double layer capacitance of OMC compensating the potential change at the ion-selective membrane/solution interface during the measurements under the control of a constant applied potential. Under the optimized conditions, the coulometric signal for the OMC-based solid-contact $\mathrm{NO}_{3}^{-}$-ISE shows two linear responses in the activity range of $1.0 \times 10^{-6}-8.0 \times 10^{-6} \mathrm{M}$ and $8.0 \times 10^{-6}-8.0 \times 10^{-4} \mathrm{M}$, and the detection limit is $4.0 \times 10^{-7} \mathrm{M}(3 \sigma / \mathrm{s})$. The proposed coulometric response also shows excellent reproducibility and stability in the presence of $\mathrm{O}_{2}$ and $\mathrm{CO}_{2}$ and light on/off. Additionally, the coulometric response shows acceptable and reliable results for detection of $\mathrm{NO}_{3}^{-}$in mineral water as compared to the traditional potentiometric response and the ion chromatography. This work provides a promising alternative signal readout for detection of ions by using solid-contact ion-selective electrodes.
\end{abstract}

(C) 2020 Elsevier B.V. All rights reserved.

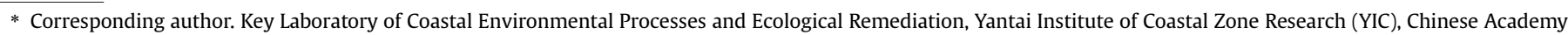
of Sciences (CAS), Shandong Provincial Key Laboratory of Coastal Environmental Processes, YICCAS, Yantai, Shandong, 264003, PR China.

** Corresponding author. School of Chemistry and Materials Science, Ludong University, Yantai, 264025, Shandong, PR China.

E-mail addresses: bqyuan@ldu.edu.cn (B. Yuan), tjyin@yic.ac.cn (T. Yin).
} 


\section{Introduction}

Nitrate is one of the essential nutrients for the growth of phytoplankton, and it is also one of the critical parameters for nitrogen biogeochemical transformations [1]. However, due to the overuse of nitrate fertilizers in agriculture and leaking from septic tanks and sewage, large amounts of nutrients containing nitrate have been discarded, which cause nitrate contamination, especially in the ground water. It's reported that the excessive exposure to the drinking water contain nitrate will cause the shortness of breath and blue-baby syndrome for infants [2]. Moreover, nitrate is also easily reduced to carcinogenic nitrite by microorganisms [3], which subsequently influence the human health. According to the regulation of U.S. Environmental Protection Agency (EPA), the maximum allowable contamination level for nitrate in drinking water is $10 \mathrm{ppm}$ (about $1.6 \times 10^{-4} \mathrm{M}$ ) [4]. Therefore, the determination of nitrate is essential for evaluating the water quality of groundwater, drinking water and reservoirs.

Spectrophotometry [5-7], ion chromatography [8,9] and electrochemical methods [10-13] are three commonly used techniques for detection of nitrate. However, both of the first two methods suffer from narrow linear range (normally less than an order of magnitude), the expensive equipment and complex operation. Potentiometric ion-selective electrodes (ISEs), as one kind of electrochemical sensors, is a promising tool for directly determining ions [14-16], due to their attractive features including wide linear range (about several orders of magnitude), free from the interferences of color and turbidity, low cost, low energy consumption and simple operation. Presently, three kinds of ISEs including liquid-contact ISEs with inner filling solutions [17-19], solidcontact ISEs [20-22] and ISEs with a functionalized conducting polymer as sensing membrane [23-25] have been reported for detection of nitrate. However, these reported $\mathrm{NO}_{3}^{-}$-ISEs suffer from a fundamental limitation of potentiometric ISEs [26], that is, the slope is confined to $59.1 \mathrm{mV} / \mathrm{dec}$ at $25^{\circ} \mathrm{C}$, due to the relationship between the potential and the logarithmic of ionic activity in the Nernst equation. This means that $4 \%$ deviation in the activity of $\mathrm{NO}_{3}^{-}$ would be obtained by the potential change of $1 \mathrm{mV}$, and the frequent calibration step is essential for accurate detection of nitrate by using the $\mathrm{NO}_{3}^{-}$-ISEs with the inadequate stability and reproducibility of the standard potential $\left(E^{0}\right)$. Therefore, in order to break through the slope limitation, the development of the nonconventional $\mathrm{NO}_{3}^{-}$-ISEs with less calibration/calibration-free procedure is highly necessary for detection of $\mathrm{NO}_{3}^{-}$in the field of environmental monitoring, agriculture and industrial analysis.

Recently, potentiometric ISEs with new readout principles through transforming the potential change of ion-selective membrane into coulometric [27], colorimetric [28], fluorimetric signal [29], optical signal of light-emitting diodes [30] and photoelectric current [31], have attracted much attention. Among them, constant-potential coulometric signal readout of solid-contact ionselective electrode with conducting polymer as solid contact, proposed by Bobacka's group [26,27,32-34], is a promising method for determining the small activity changes of the primary ion in solution. The mechanism for obtaining the coulometric signal is based on the redox capacitance of conducting polymer-based solid contact involving both the reversible oxidation of the conjugated polymer chains and the insertion/expulsion of chargecompensating ions, which convert a change in ion concentration (activity) into a transient current pulse and the corresponding integrated charge at a constant potential [27,32]. However, it's reported that conducting polymer-based solid contact with redox capacitance may suffer from some undesirable side reactions caused by the presence of $\mathrm{O}_{2}, \mathrm{CO}_{2}$ and $\mathrm{H}_{2} \mathrm{O}$ during the process of ion-to-electron transduction between the electronic conductor and ion-selective membrane [16], which subsequently cause a bias in the coulometric response of the solid-contact ISE [26]. In fact, another kind of solid contacts with electrical double layer capacitance, such as fullerence [35], carbon nanotubes [36], graphene [37], three-dimensionally ordered macroporous carbon [38], colloid imprinted mesoporous carbon [39] and nanoporous gold [40] have been widely reported for developing stable solid-contact ISEs. The transduction mechanism of solid contacts with electrical double layer capacitance between the ion-selective membrane and electronic conductor depends on the quantity of ionic charge from the ion-selective membrane and electrical charge in the electrical double layer, rather than the redox reaction for the solid contact [41]. Therefore, the performance of solid contacts with electrical double layer capacitance would not be influenced by chemical redox reactions (e.g. due to $\mathrm{O}_{2}$ ). However, as far as we know, there are few reports about obtaining the coulometric signal based on solid contact with electrical double layer capacitance by using the solid-contact ISEs.

Herein, ordered mesoporous carbon (OMC) material with electrical double layer capacitance was chosen as a model to develop the solid-contact $\mathrm{NO}_{3}^{-}$-ISE, and the corresponding coulometric signal performance was investigated. The influence of the amount of OMC-based solid contact, the type of polymer matrix, the presence of $\mathrm{O}_{2}, \mathrm{CO}_{2}$ and light on the coulometric signal was also characterized.

\section{Experimental}

\subsection{Reagents and materials}

Poly(vinyl chloride) (PVC), bis(2-ethylhexyl)sebacate (DOS), poly(methyl methacrylate-co-buty methacrylate) (MMA-BMA), polyurethane (PU), tridodecylmethylammonium nitrate (TDMA$\mathrm{NO}_{3}^{-}$) and tetradodecylammonium tetrakis(4-chlorophenyl)borate (ETH 500) were purchased from Sigma-Aldrich. Room temperature vulcanizing-type silicone rubber (SR, RTV 3140) was obtained from Dow Corning. Ordered mesoporous carbon (OMC) with average pore diameter of $3.8-4 \mathrm{~nm}$ and BET surface area $\geq 900 \mathrm{~m}^{2} / \mathrm{g}$ was obtained from Nanjing XFNANO Materials Tech Co., Ltd. Freshly distilled tetrahydrofuran (THF) was used throughout the experiment. All other chemicals with analytical reagent grade were used. Aqueous solutions were prepared by deionized water $(18.2 \mathrm{M} \Omega \mathrm{cm}$ specific resistance) obtained with a Pall Cascada laboratory water system.

\subsection{Preparation of solid-contact $\mathrm{NO}_{3}^{-}$-ISEs}

The GC disk electrode ( $3 \mathrm{~mm}$ in diameter) was polished with $0.05 \mu \mathrm{m} \mathrm{Al}{ }_{2} \mathrm{O}_{3}$ slurry, then ultrasonically cleaned and washed with ethanol and deionized water. The solid-contact suspension was prepared by ultrasonicating $3 \mathrm{mg}$ OMC material in $1 \mathrm{ml}$ THF for $30 \mathrm{~min}$, and then drop cast on the polished GC electrode. After being dried at room temperature, the OMC modified GC electrode (GC/OMC) was obtained for further use.

In the experimental, two kinds of $\mathrm{NO}_{3}^{-}$-selective membranes were used. One kind of $\mathrm{NO}_{3}^{-}$-selective membrane components contain 1 wt\% TDMA-NO- ${ }_{3}^{-}, 1$ wt\% ETH 500, 65.3 wt\% DOS and 32.7 wt\% polymer matrix (PVC, PU or SR), and another kind of $\mathrm{NO}_{3}^{-}-$ selective membrane components contain $1 \mathrm{wt} \% \mathrm{TDMA}^{-\mathrm{NO}_{3}^{-}}, 1 \mathrm{wt} \%$ ETH 500 and 98 wt\% MMA-BMA. The membrane cocktails were separately prepared by dissolving $100 \mathrm{mg}$ of the membrane components in $1 \mathrm{ml}$ THF and then stirred for at least $2 \mathrm{~h}$ in order to obtain the homogeneous solutions. Then $15 \mu$ l of the membrane cocktails were drop cast on the above GC/OMC electrodes, respectively. After being dried at room temperature, the prepared 
electrodes, which were denoted as $\mathrm{GC} / \mathrm{OMC} / \mathrm{NO}_{3}^{-}$-ISE, were conditioned in $1.0 \times 10^{-3} \mathrm{M} \mathrm{NaNO}_{3}$ overnight. For comparison, the coated-disk $\mathrm{NO}_{3}^{-}$-ISE (GC/NO- -ISE) was prepared by directly coating the $\mathrm{NO}_{3}^{-}$-selective membrane cocktail on the bare $\mathrm{GC}$ electrode.

\subsection{Apparatus and measurements}

Potentiometric measurements were carried out by using $\mathrm{CHI}$ 760D electrochemical workstation (Shanghai Chenhua Apparatus) at room temperature with $\mathrm{Ag} / \mathrm{AgCl}(3 \mathrm{M} \mathrm{KCl})$ electrode as a reference electrode in the galvanic cell: $\mathrm{Ag}|\mathrm{AgCl}(3 \mathrm{M} \mathrm{KCl})|$ sample solution $\mid \mathrm{NO}_{3}^{-}$-selective membrane|OMC|GC electrode. The liquid junction potentials and ion activities were calculated by the Henderson and Debye-Hückel equations, respectively.

Chronoamperometric measurements were performed under stirring with a three-electrode system, where the $\mathrm{GC} / \mathrm{OMC} / \mathrm{NO}_{3}^{-}$-ISE was used as a working electrode, an $\mathrm{Ag} / \mathrm{AgCl}$ ( $3 \mathrm{M} \mathrm{KCl}$ ) as a reference electrode and a platinum wire as an auxiliary electrode. Before the measurements, the proposed solid-contact $\mathrm{NO}_{3}^{-}$-ISE was allowed to equilibrate for at least $10 \mathrm{~min}$ in deionized water under the control of the open circuit potential in order to reduce the background drift and obtain a stable current with the order of magnitude of $1 \mathrm{nA}$. Then, the solution containing $\mathrm{NO}_{3}^{-}$was added in deionized water, and a transient current signal was subsequently obtained. Finally, considering the analytical time for the practical application, the corresponding integrated charge $(\mathrm{Q})$ was estimated at a time interval of $500 \mathrm{~s}$ through integrating the measured current-time curve. Electrochemical impedance spectroscopy was also measured with the above three-electrode system in $0.1 \mathrm{M} \mathrm{KCl}$ in order to compare the impedance performance among the different kinds of polymeric matrix-based solid-contact $\mathrm{NO}_{3}^{-}$-selective membrane. A Dionex ICS-3000 ion chromatography (Sunnyvale, CA, USA) system was used for detecting $\mathrm{NO}_{3}^{-}$in mineral water samples.

\section{Results and discussion}

\subsection{Influence of solid contact and polymer matrix on the coulometric signal}

Unlike conducting polymer-based solid contacts with redox capacitance, solid contact based on electrical double layer capacitance plays a role in compensating the potential change at the ionselective membrane/solution interface through adjusting the quantity of ionic charge from ion-selective membrane and electrical charge in the electrical double layer during the measurements of coulometric signal at the constant applied potential (Fig. 1). Therefore, the influence of the charge capacity, which is related to the electrical double layer capacitance of solid contact on the coulometric signal, needs to be investigated. As shown in Fig. 2, all of the capacitive currents of OMC modified GC electrodes are found to be larger than that of the bare GC electrode, indicating that the electrical double layer capacitance of OMC-based solid contacts is larger than that of the bare GC electrode, due to the large specific surface area and excellent conductivity for OMC materials. Additionally, the electrical double layer capacitance of OMC modified GC electrodes are observed to be increased as the increase in the amount of OMC, which is consistent with that demonstrated by electrochemical impedance spectroscopy (Fig. S1). Moreover, all of the cyclic voltammograms for the OMC modified GC electrode show two region characteristics. One is related to the redox process caused by the oxygen reduction from -0.5 to $0 \mathrm{~V}$, and the other is related to the capacitive process with a near-rectangular shape from 0 to $0.5 \mathrm{~V}$. Similar behaviors were also found for carbon nanotubes and graphene based solid contact [42,43], while there are slight differences from the our previous work [44], which may be due to the different thickness of the OMC-based solid contact.

The influence of the amounts of OMC-based solid contact on the coulometric signal obtained by the solid-contact $\mathrm{NO}_{3}^{-}$-ISEs was investigated. As shown in Fig. 3a, a transient current associated with the quantity of charge of the OMC-based solid contact is observed upon the addition of $\mathrm{NO}_{3}^{-}$under the control of open circuit potential, and the decay time is found to be increased with the amount of OMC. Additionally, the corresponding integrated charge is also found to be increased with the amount of OMC (Fig. 3b). These phenomena are consistent with the coulometric signal obtained by the reported conducting polymer-based solid contact [27,34]. Considering the analytical time for the practical application, $2 \mu \mathrm{l}$ of the OMC solution was chosen for the further use.

On the other hand, the transient current is also reported to be related to the resistance of the electrochemical cell [34], especially for the ion-selective membrane. It's known that the types of polymer matrix also influence the resistance of ion-selective the membrane, beside the thickness of the membrane. Therefore, the influence of the types of polymer matrix on the coulometric signal obtained by the solid-contact $\mathrm{NO}_{3}^{-}$-ISEs was investigated. In the experimental, four types of polymer matrix, including PVC, PU, SR and MMA-BMA, have been used to prepare the $\mathrm{NO}_{3}^{-}$-selective membrane. As shown in Fig. 4, the PVC-based ion-selective membrane shows the highest transient current and the corresponding integrated charge as compared to the other types of polymer matrix. The reason may be due to the fact that the resistance of PVCbased ion-selective membrane is smallest than other types of polymer matrix (Fig. S2). Additionally, it's reported that the thin ion-selective membrane shows a low resistance, and is favor for obtaining fast response time due to the effective mass transport across the membrane triggered by the transient current $[26,34]$. Therefore, in this manuscript, $15 \mu \mathrm{l}$ of the ion-selective membrane cocktail was chosen, which just covered the OMC modified glassy carbon electrode.

\subsection{Coulometric signal performance of the OMC-based solid- contact $\mathrm{NO}_{3}^{-}-\mathrm{ISE}$}

The coulometric signal for the proposed OMC-based solid-contact $\mathrm{NO}_{3}^{-}$-ISE was measured by increasing the activity of $\mathrm{NO}_{3}^{-}$, which is different from the reported dilution method for obtaining the coulometric signal by using the conducting polymer-based solid-contact ISEs [26,27,32-34]. Before each measurement, the electrode was equilibrated for at least $10 \mathrm{~min}$ under the control of the open circuit potential in deionized water in order to obtain a stable current with the order of magnitude of $1 \mathrm{nA}$. As shown in Fig. 5, a transient current is observed through each change of $\mathrm{NO}_{3}^{-}$ activity under the optimized condition, which is originated from the potential change at the solution/membrane surface and the potential compensation of OMC-based solid contact under the control of the constant potential. Moreover, the transient current and the corresponding integrated charge are found to be increased as the increase in the $\mathrm{NO}_{3}^{-}$activity (Fig. $5 \mathrm{a}$ and b). The coulometric signal for the OMC-based solid-contact $\mathrm{NO}_{3}^{-}$-ISE show two linear responses in the activity range of $1.0 \times 10^{-6}-8.0 \times 10^{-6} \mathrm{M}$ and $8.0 \times 10^{-6}-8.0 \times 10^{-4} \mathrm{M}$, respectively (Fig. $5 \mathrm{c}$ ). The linear equations between the integrated charge and logarithmic of ionic activity are shown as follows: $Q=6.85 \log a+43.42(R=0.9963, n=3)$ and $Q=$ $9.56 \log a+56.55(R=0.9981, n=3)$, respectively. The detection limit is calculated to be $4.0 \times 10^{-7} \mathrm{M}(3 \sigma / \mathrm{s})$. Additionally, the coulometry signal is found to show a lower detection limit, as compared to the potential response for the OMC-based solid-contact $\mathrm{NO}_{3}^{-}$-ISE at the zero-current condition (Fig. 5d), which shows a 


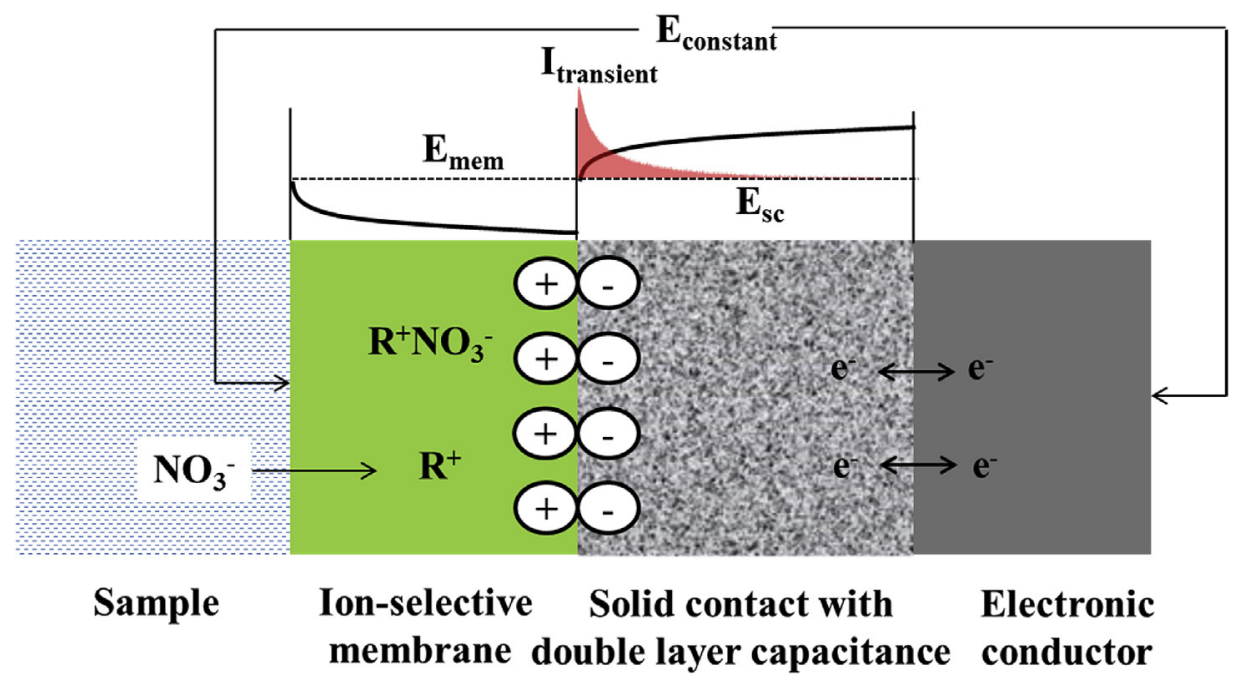

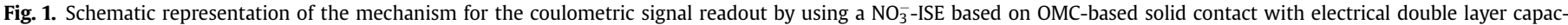

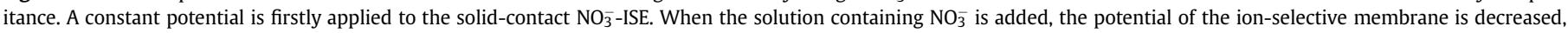

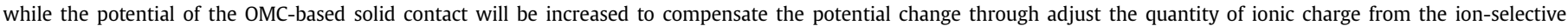
membrane and electrical charge in solid contact. This process will produce a transient current until a new equilibrium state is obtained.

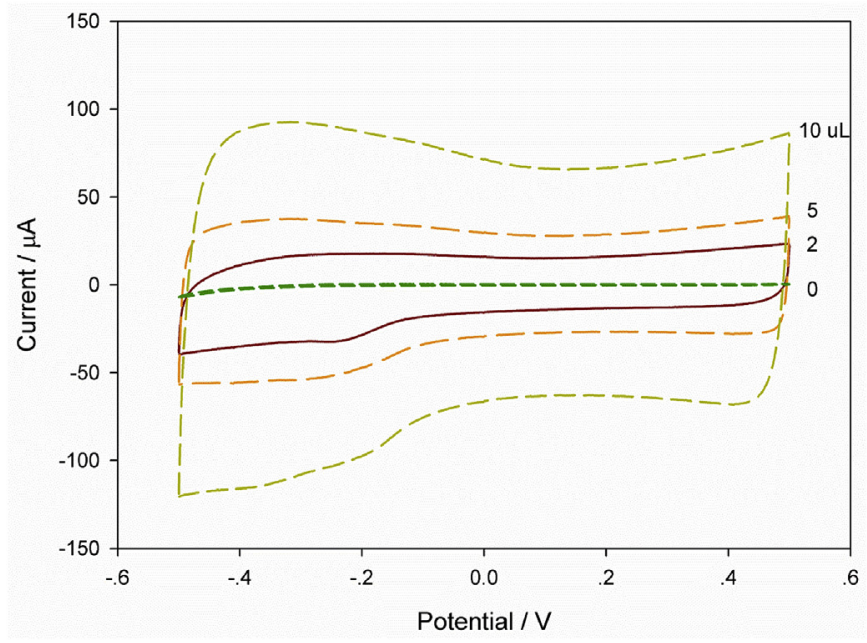

Fig. 2. Cyclic voltammograms for the bare GC electrode and the modified GC electrodes with different amounts of ordered mesoporous carbon material in $0.1 \mathrm{M} \mathrm{KCl}$. Scan rate $=100 \mathrm{mV} / \mathrm{s}$. linear response in the range of $4.0 \times 10^{-6}-8.0 \times 10^{-4} \mathrm{M}$ with the slope of $61.4 \mathrm{mV} / \mathrm{dec}$. However, the response time for the coulometry signal is much longer than that of the potential response (Fig. S3), which may be due to that the mass transport process across the ion-selective membrane triggered by the transient current limits the time response [34]. Moreover, the stability of the OMC-based solid-contact $\mathrm{NO}_{3}^{-}$-ISE was investigated by repeatedly measuring the coulometric signal upon the addition of $10^{-4} \mathrm{M} \mathrm{NO}_{3}^{-}$. As shown in Fig. 6, both the transient current and the corresponding integrated charge show reproducible responses, which is attributed to the high electrical power and outstanding cycle life of OMC with large specific surface area and interconnected pores when acting as electrochemical double-layer supercapacitor [45].

The influence of $\mathrm{O}_{2}, \mathrm{CO}_{2}$ and light on the coulometric signal of OMC-based solid-contact $\mathrm{NO}_{3}^{-}$-ISE were investigated through turning on/off the light and purging gas in the aqueous solutions, respectively. As shown in Fig. 7a, there are no significant changes in the transient current and the corresponding integrated charge in the presence of $\mathrm{O}_{2}$ and $\mathrm{CO}_{2}$, indicating that no undesirable effect caused by the permeation of $\mathrm{O}_{2}$ and $\mathrm{CO}_{2}$ into the ion-selective membrane occur. Moreover, no obvious change in the coulometric signal for the OMC-based solid-contact ISE was observed during the light on/off, which is quite different from conducting

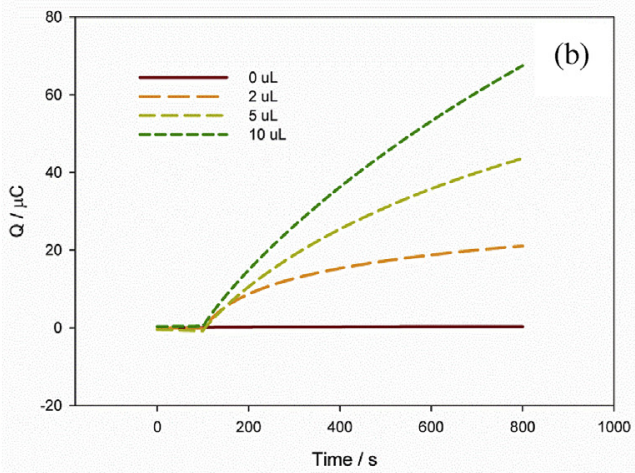

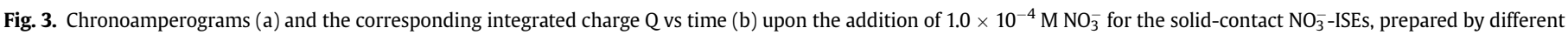
amounts of ordered mesoporous carbon material as solid contact. 

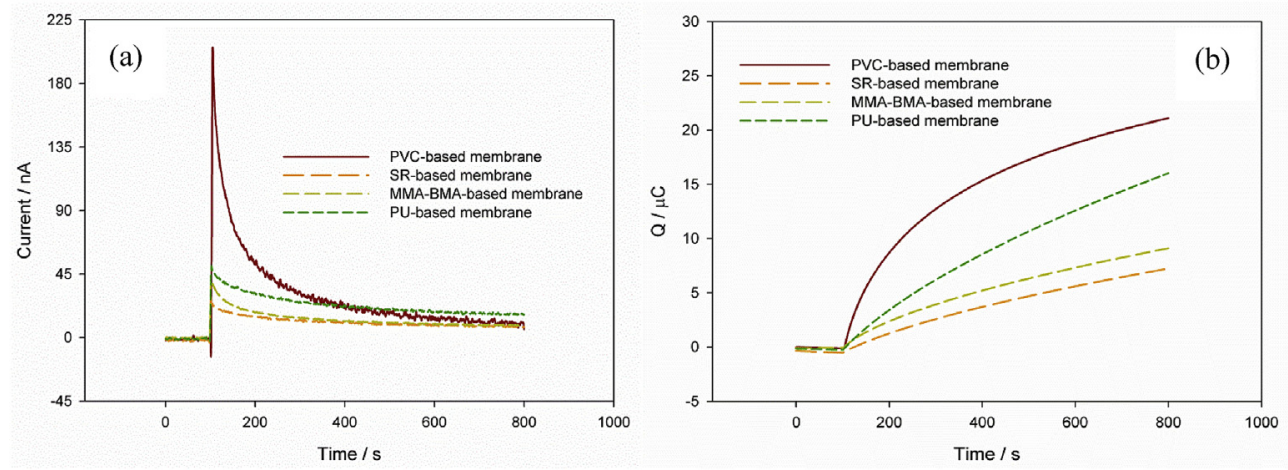

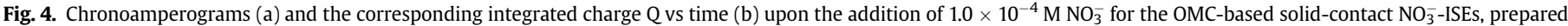
by different types of polymeric matrix.
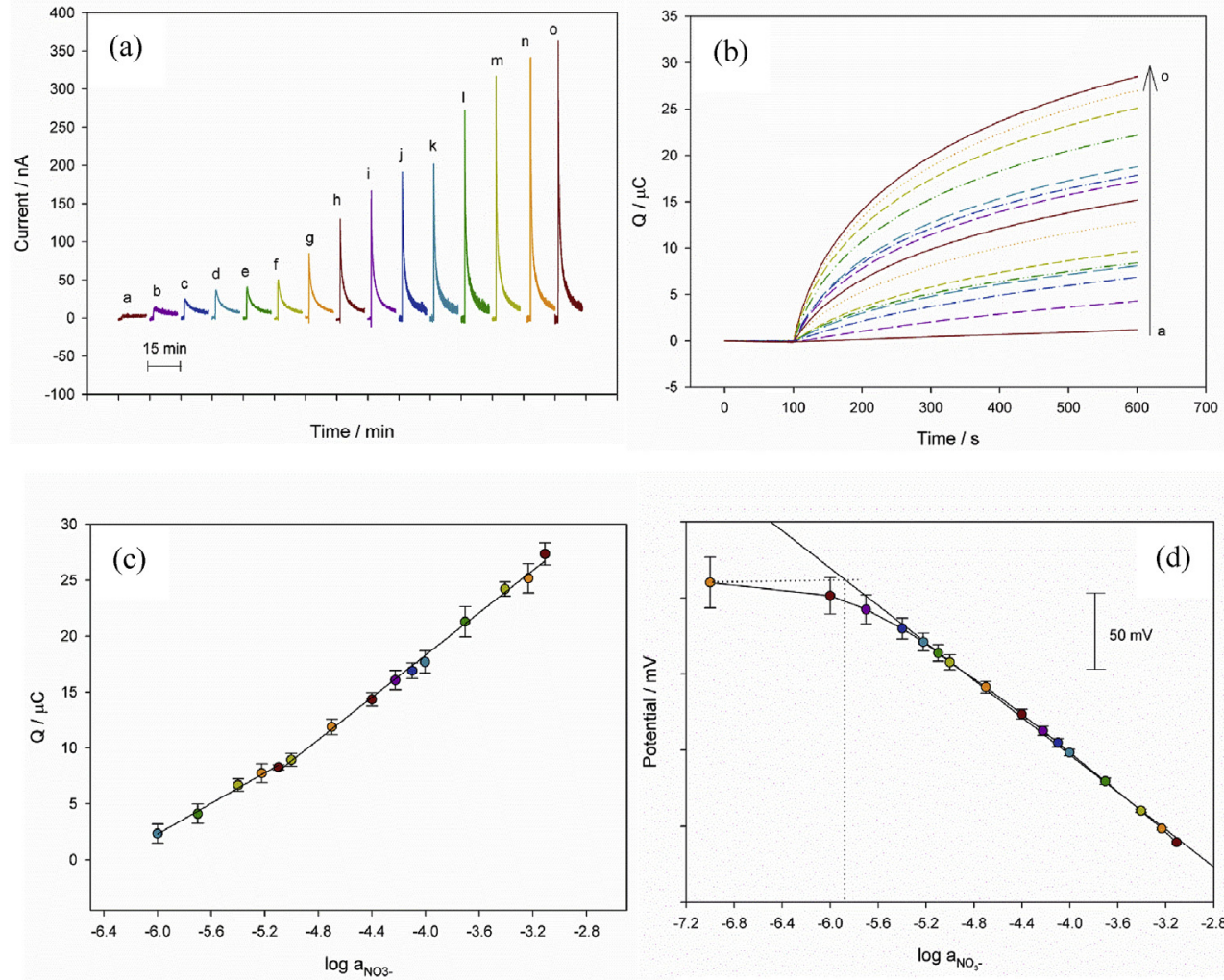

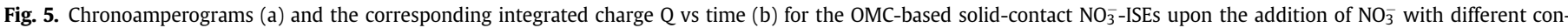

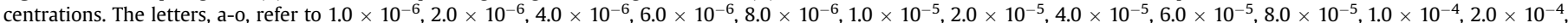

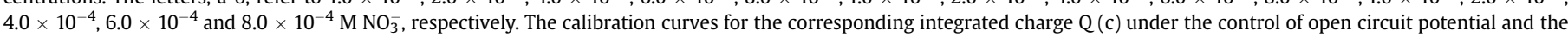

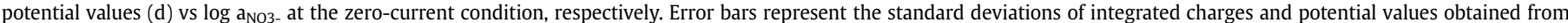
three measurements.

polymer-based solid-contact ISE [46]. These results indicate that the coulometric response for the solid-contact ISE prepared by OMC as solid contact is stable in the presence of $\mathrm{O}_{2}$ and $\mathrm{CO}_{2}$ and light on/off. Additionally, the selectivity of the coulometric signal for the OMC-based solid-contact ISE was also investigated. As shown in Fig. 7b, as the increase in the concentration of $\mathrm{NaCl}$, both the transient current and the corresponding integrated charge are found to be decreased. This phenomenon is consistent with the potential response of OMC-based solid-contact ISE (Fig. S4), which indicates that the coulometric signal for the OMC-based solidcontact ISE depends on the inherent selectivity of the $\mathrm{NO}_{3}^{-}$-selective membrane $\left(\log \mathrm{K}_{\overline{\mathrm{N}} \mathrm{O}}, \overline{\mathrm{Cl}}=-2.0\right.$, estimated by separated solution method in Fig. S5).

\subsection{Practical application}

In order to demonstrate the analytical applications for the coulometric signal, the OMC-based solid-contact ISE was tested by measuring $\mathrm{NO}_{3}^{-}$in mineral water samples through the standard addition method in order to eliminate the influence of the background electrolyte. The detection procedure includes: (1) to obtain the stable baseline through equilibrating the proposed solidcontact $\mathrm{NO}_{3}^{-}$-ISE in mineral water under the control of the open circuit potential, (2) to add a certain volume of a standard solution 


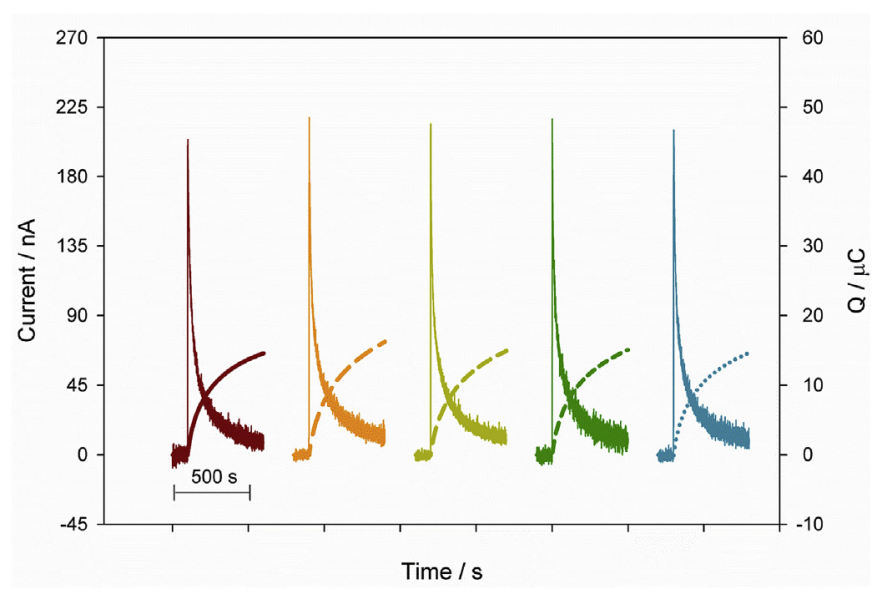

Fig. 6. Reproducibility of the chronoamperograms and the corresponding integrated charge $Q$ vs time upon the addition of $1.0 \times 10^{-4} \mathrm{M} \mathrm{NO}_{3}^{-}$for the OMC-based solidcontact $\mathrm{NO}_{3}^{-}$-ISEs.

containing $\mathrm{NO}_{3}^{-}$in mineral water, which may lead to a transient current, (3) to obtain the corresponding integrated charge (Q1) through integrating the transient current-time curve, (4) to add another volume of the standard solution containing $\mathrm{NO}_{3}^{-}$and to obtain the another integrated charge (Q2) though repeating the steps (1)-(3). Finally, the concentration of nitrate in mineral water samples was calculated according to the following equation [34]:

$Q_{p}=C_{c p} \frac{s}{z_{i}} \log \left(\frac{a_{i}(\text { initial })}{a_{i}(\text { final })}\right)$

Where, $C_{c p}$ is the capacitance of the OMC-based solid contact, $s$ is the slope, $z_{i}$ is the charge of the analyte ion (here, $z_{i}$ is -1 for $\mathrm{NO}_{3}^{-}$). Additionally, for comparison, the concentrations of $\mathrm{NO}_{3}^{-}$in mineral water were also measured by the traditional potentiometric response at zero-current condition with the standard addition method. As shown in Table 1, the results detected by the coulometric signal are comparable to those obtained by the traditional potentiometric response and the ion chromatography, which indicates the coulometric signal for the solid-contact $\mathrm{NO}_{3}^{-}$-ISE is reliable and acceptable.

\section{Conclusions}

As compared to the traditional potential response at the zerocurrent condition, a coulometric signal is proposed for detection of $\mathrm{NO}_{3}^{-}$based on an ordered mesoporous carbon-based solid-
Table 1

Comparisons of the results for determination of $\mathrm{NO}_{3}^{-}$in mineral water samples by using the proposed OMC-based solid-contact $\mathrm{NO}_{3}^{-}$with the coulometric signal under the control of the constant potential and potential response at the zero-current condition and ion chromatography.

\begin{tabular}{|c|c|c|c|}
\hline \multirow[t]{2}{*}{ Sample } & Coulometric signal & Potential response & Ion chromatography \\
\hline & Found $^{\mathrm{a}}(\mathrm{M})$ & Found $^{\mathrm{a}}(\mathrm{M})$ & Found $^{\mathrm{a}}(\mathrm{M})$ \\
\hline 1 & $(2.72 \pm 0.23) \times 10^{-5}$ & $(2.77 \pm 0.22) \times 10^{-5}$ & $(2.71 \pm 0.12) \times 10^{-5}$ \\
\hline 2 & $(5.44 \pm 0.29) \times 10^{-5}$ & $(5.49 \pm 0.22) \times 10^{-5}$ & $(5.49 \pm 0.49) \times 10^{-5}$ \\
\hline 3 & $(6.43 \pm 0.17) \times 10^{-5}$ & $(6.37 \pm 0.76) \times 10^{-5}$ & $(6.40 \pm 0.48) \times 10^{-5}$ \\
\hline 4 & $(2.23 \pm 0.39) \times 10^{-5}$ & $(2.33 \pm 0.19) \times 10^{-5}$ & $(2.29 \pm 0.12) \times 10^{-5}$ \\
\hline 5 & $(5.51 \pm 0.17) \times 10^{-5}$ & $(5.52 \pm 0.93) \times 10^{-5}$ & $(5.32 \pm 0.15) \times 10^{-5}$ \\
\hline
\end{tabular}

a Mean of three measurements \pm standard deviation.

contact ion-selective electrode under the control of a constant potential. The mechanism is based on the potential change caused by the addition of $\mathrm{NO}_{3}^{-}$at the interface between the ion-selective membrane and the testing solution and subsequently potential change of OMC-based solid contact associated with the change in transient current. Through integrating the transient current-time curves for each $\mathrm{NO}_{3}^{-}$activity, the corresponding charge is found to be proportional to the logarithm of ion activity in the range of $1.0 \times 10^{-6}-8.0 \times 10^{-6} \mathrm{M}$ and $8.0 \times 10^{-6}-8.0 \times 10^{-4} \mathrm{M}$, respectively. Additionally, it's found that the electrical double layer capacitance of OMC-based solid contact has the same ability to obtain the transient current and the corresponding accumulated charge as the redox capacitance of conducting polymer-based solid contact, but also the OMC-based solid-contact ISE shows excellent reproducibility and stability in the presence of $\mathrm{O}_{2}$ and $\mathrm{CO}_{2}$ and light on/off during the measurement of coulometric response. This work provides an alternative signal readout for detection of ions by using solid-contact ion-selective electrodes, which is promising for breaking through the slope limitation of potentiometric ion sensors, due to the relationship between the potential and the logarithmic of ionic activity in the Nernst equation.

\section{CRediT authorship contribution statement}

Hemin Wang: Conceptualization, Methodology, Validation, Investigation, Data curation, Writing - original draft, Writing - review \& editing. Baiqing Yuan: Formal analysis, Writing - review \& editing, Supervision, Visualization. Tanji Yin: Conceptualization, Methodology, Validation, Formal analysis, Investigation, Resources, Data curation, Writing - original draft, Writing - review \& editing, Visualization, Supervision, Project administration, Funding acquisition. Wei Qin: Formal analysis, Resources, Writing - original draft, Writing - review \& editing, Visualization, Project administration, Funding acquisition.
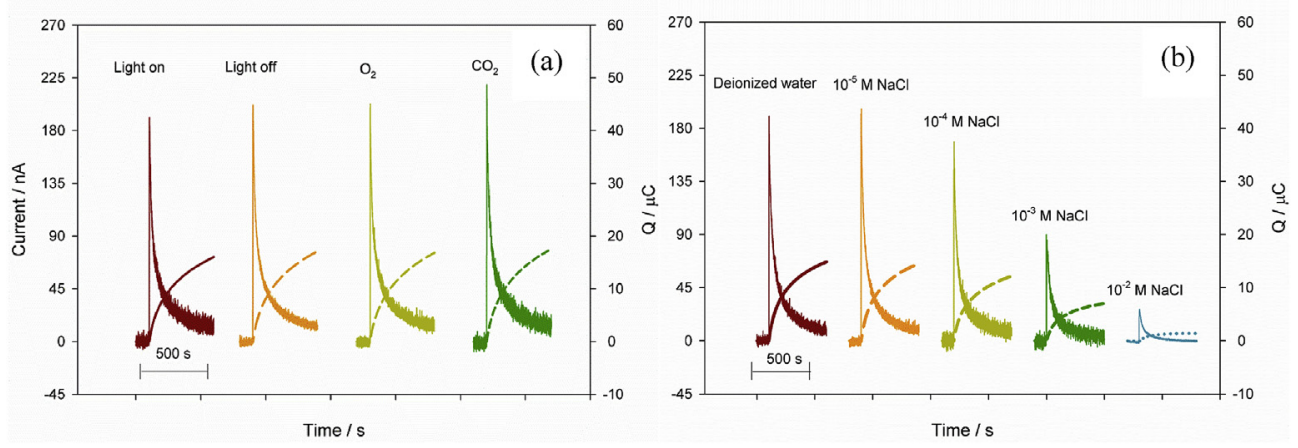

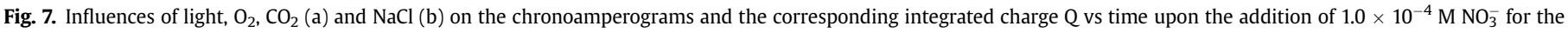
OMC-based solid-contact $\mathrm{NO}_{3}^{-}$-ISEs. The used concentration of $\mathrm{O}_{2}$ and $\mathrm{CO}_{2}$ is saturated in the aqueous solution at room temperature. 


\section{Declaration of competing interest}

The authors declare that they have no known competing financial interests or personal relationships that could have appeared to influence the work reported in this paper.

\section{Acknowledgments}

This research was financially supported by the National Natural Science Foundation of China (41706110, 21677172), National Key Research and Development Program of China (2016YFC1400700), Taishan Scholar Program of Shandong Province (TSPD20181215), and Key Laboratory of Coastal Environmental Processes and Ecological Remediation, YICCAS (2018KFJJ03).

\section{Appendix A. Supplementary data}

Supplementary data to this article can be found online at https://doi.org/10.1016/j.aca.2020.07.019.

\section{References}

[1] K. Lin, P. Li, J. Ma, D. Yuan, An automatic reserve flow injection method using vanadium (III) reduction for simultaneous determination of nitrite and nitrate in estuarine and coastal waters, Talanta 195 (2019) 613-618.

[2] M. Dozier, R. Melton, M. Hare, J. Hopkins, B.J. Lesikar, Drinking water problems: nitrates, Available electronically from, http://hdl.handle.net/1969.1/ 87342, 2008.

[3] J.O. Lundberg, E. Weitzberg, J.A. Cole, N. Benjamin, Nitrate, bacteria and human health, Nat. Rev. Microbiol. 2 (2004) 593-602.

[4] U.S. Environmental Protection Agency. https://www.epa.gov/ground-waterand-drinking-water/national-primary-drinking-water-regulations.

[5] T. Fang, P. Li, K. Lin, N. Chen, Y. Jiang, J. Chen, D. Yuan, J. Ma, Simultaneous underway analysis of nitrate and nitrite in estuarine and coastal waters using an automated integrated syringe-pump-based environmental-water analyzer, Anal. Chim. Acta 1076 (2019) 100-109.

[6] A. Kazemzadeh, A.A. Ensafi, Simultaneous determination of nitrite and nitrate in various samples using flow-injection spectrophotometric detection, Microchem. J. 69 (2001) 159-166.

[7] J.M. Wang, J.C. Zhang, Z.J. Zhang, Rapid determination of nitrate nitrogen and nitrite nitrogen by second derivative spectrophotometry, Spectrosc. Spectr. Anal. 39 (2019) 161-165.

[8] C. Lopez-Moreno, I.V. Perez, A.M. Urbano, Development and validation of an ionic chromatography method for the determination of nitrate, nitrite and chloride in meat, Food Chem. 194 (2016) 687-694.

[9] J. Yang, Y. Kwon, M. Duca, M.T.M. Koper, Combining voltammetry and ion chromatography: application to the selective reduction of nitrate on Pt and PtSn electrodes, Anal. Chem. 85 (2013) 7645-7649.

[10] M. Cuartero, G.A. Crespo, E. Bakker, Tandem electrochemical desalinationpotentiometric nitrate sensing for seawater analysis, Anal. Chem. 87 (2015) 8084-8089.

[11] W. Ren, S. Mura, J.M.K. Irudayaraj, Modified graphene oxide sensors for ultrasensitive detection of nitrate ions in water, Talanta 143 (2015) 234-239.

[12] W. Tang, J. Ping, K. Fan, Y. Wang, X. Luo, Y. Ying, J. Wu, Q. Zhou, All-solid-state nitrate-selective electrode and its application in drinking water, Electrochim. Acta 81 (2012) 186-190.

[13] M.E.E. Alahi, S.C. Mukhopadhyay, Detection methods of nitrate in water: a review, Sens. Actuator A-Phys. 280 (2018) 210-221.

[14] G.A. Crespo, Recent advances in ion-selective membrane electrodes for in situ environmental water analysis, Electrochim. Acta 245 (2017) 1023-1034.

[15] E. Bakker, Electroanalysis with membrane electrodes and liquid-liquid interfaces, Anal. Chem. 88 (2016) 395-413.

[16] J. Bobacka, A. Ivaska, A. Lewenstam, Potentiometric ion sensors, Chem. Rev. 108 (2008) 329-351.

[17] T. Le Goff, J. Braven, L. Ebdon, N.P. Chilcott, D. Scholefield, J.W. Wood, An accurate and stable nitrate-selective electrode for the in situ determination of nitrate in agricultural drainage waters, Analyst 127 (2002) 507-511.

[18] A.S. Watts, V.G. Gavalas, A. Cammers, P.S. Andrada, M. Alajarin, L.G. Bachas, Nitrate-selective electrode based on a cyclic bis-thiourea ionophore, Sensor. Actuator. B Chem. 121 (2007) 200-207.

[19] P.G. Sutton, J. Braven, L. Ebdon, D. Scholefield, Development of a sensitive nitrate-selective electrode for on-site use in fresh waters, Analyst 124 (1999)
$877-882$.

20] W.Z. Tang, J.F. Ping, K. Fan, Y.X. Wang, X. Luo, Y.B. Ying, J. Wu, Q.L. Zhou, Allsolid-state nitrate-selective electrode and its application in drinking water Electrochim. Acta 81 (2012) 186-190.

[21] B. Paczosa-Bator, Effects of type of nanosized carbon black on the performance of an all-solid-state potentiometric electrode for nitrate, Microchim. Acta 181 (2014) 1093-1099.

[22] B. Paczosa-Bator, L. Cabaj, R. Piech, K. Skupien, Potentiometric sensors with carbon black supporting platinum nanoparticles, Anal. Chem. 85 (2013) $10255-10261$.

[23] T.A. Bendikov, J. Kim, T.C. Harmon, Development and environmental application of a nitrate selective microsensor based on doped polypyrrole films, Sensor. Actuator. B Chem. 106 (2005) 512-517.

[24] T.A. Bendikov, S. Miserendino, Y.C. Tai, T.C. Harmon, A parylene-protected nitrate selective microsensor on a carbon fiber cross section, Sensor. Actuator. B Chem. 123 (2007) 127-134.

[25] S. Aravamudhan, S. Bhansali, Development of micro-fluidic nitrate-selective sensor based on doped-polypyrrole nanowires, Sensor. Actuator. B Chem. 132 (2008) 623-630.

[26] T. Han, U. Mattinen, J. Bobacka, Improving the sensitivity of solid-contact ionselective electrodes by using coulometric signal transduction, ACS Sens. 4 (2019) 900-906.

[27] U. Vanamo, E. Hupa, V. Yrjänä, J. Bobacka, New signal readout principle for solid-contact ion-selective electrodes, Anal. Chem. 88 (2016) 4369-4374.

[28] S. Jansod, M. Cuartero, T. Cherubini, E. Bakker, Colorimetric readout for potentiometric sensors with closed bipolar electrodes, Anal. Chem. 90 (2018) 6376-6379.

[29] E. Jaworska, A. Michalska, K. Maksymiuk, Fluorimetric readout of ion-selective electrode potential changes, Electrochim. Acta 284 (2018) 321-327.

[30] J. Zhai, L. Yang, X. Du, X. Xie, Electrochemical-to-optical signal transduction for ion-selective electrodes with light-emitting diodes, Anal. Chem. 90 (2018) $12791-12795$.

[31] X. Hun, X. Xiong, J. Ding, W. Qin, Photoelectric current as a highly sensitive readout for potentiometric sensors, Chem. Commun. 56 (2020) 3879-3882.

[32] E. Hupa, U. Vanamo, J. Bobacka, Novel ion-to-electron transduction principle for solid-contact ISEs, Electroanalysis 27 (2015) 591-594.

[33] T. Han, U. Vanamo, J. Bobacka, Influence of electrode geometry on the response of solid-contact ion-selective electrodes when utilizing a new coulometric signal readout method, ChemElectroChem 3 (2016) 2071-2077.

[34] Z. Jarolímová, T. Han, U. Mattinen, J. Bobacka, E. Bakker, Capacitive model for coulometric readout of ion-selective electrodes, Anal. Chem. 90 (2018) 8700-8707.

[35] M. Fouskaki, N. Chaniotakis, Fullerene-based electrochemical buffer layer for ion-selective electrodes, Analyst 133 (2008) 1072-1075.

[36] G. Crespo, D. Gugsa, S. Macho, F. Rius, Solid-contact pH-selective electrode using multi-walled carbon nanotubes, Anal. Bioanal. Chem. 395 (2009) $2371-2376$

[37] J. Ping, Y. Wang, J. Wu, Y. Ying, Development of an all-solid-state potassium ion-selective electrode using graphene as the solid-contact transducer, Electrochem. Commun. 13 (2011) 1529-1532.

[38] C.Z. Lai, M.A. Fierke, A. Stein, P. Bühlmann, Ion-selective electrodes with threedimensionally ordered macroporous carbon as the solid contact, Anal. Chem. 79 (2007) 4621-4626.

[39] J.B. Hu, X.U. Zou, A. Stein, P. Buhlmann, Ion-selective electrodes with colloidimprinted mesoporous carbon as solid contact, Anal. Chem. 86 (2014) 7111-7118.

[40] T.J. Yin, D.W. Pan, W. Qin, All-solid-state polymeric membrane ion-selective miniaturized electrodes based on a nanoporous gold film as solid contact, Anal. Chem. 86 (2014) 11038-11044.

[41] J.B. Hu, A. Stein, P. Buhlmann, Rational design of all-solid-state ion-selective electrodes and reference electrodes, Trac. Trends Anal. Chem. 76 (2016) $102-114$.

[42] G.A. Crespo, S. Macho, J. Bobacka, F.X. Rius, Transduction mechanism of carbon nanotubes in solid-contact ion-selective electrodes, Anal. Chem. 81 (2008) $676-681$.

[43] R. Hernandez, J. Riu, J. Bobacka, C. Valles, P. Jimenez, A.M. Benito, W.K. Maser, F.X. Rius, Reduced graphene oxide films as solid transducers in potentiometric all-solid-state ion-selective electrodes, J. Phys. Chem. C 116 (2012) 22570-22578.

[44] T. Yin, T. Han, C. Li, W. Qin, J. Bobacka, Real-time monitoring of the dissolution of silver nanoparticles by using a solid-contact $\mathrm{Ag}^{+}$-selective electrode, Anal. Chim. Acta 1101 (2020) 50-57.

[45] Z. Yu, L. Tetard, L. Zhai, J. Thomas, Supercapacitor electrode materials: nanostructures from 0 to 3 dimensions, Energy Environ. Sci. 8 (2015) 702-730.

[46] T. Lindfors, Light sensitivity and potential stability of electrically conducting polymers commonly used in solid contact ion-selective electrodes, J. Solid State Electrochem. 13 (2009) 77-89. 